This report was prepared as an account of work sponsored by an agency of the United States Government. Neither the United States Government nor any agency thereof, nor any of their employees, makes any warranty, express or implied, or assumes any legal liability or responsibility for the accuracy, completeness, or usefulness of any information, apparatus, product, or process disclosed, or represents that its use would not infringe privately owned rights. Reference herein to any specific commercial product, prosess. or service by trade name, trademark, manufecturer, of otherwise does not nocessarily conslitute or imply its endorsement, recommendation, or favoriag by the United States Government or any agency thereof. The vicws and opinions of authors expreased herein do not necessarily state or reflect those of the United States Goverument or any agency thereof.

\title{
Relaxation Phenomena i: the High Temperature S-1 Spheromak
}

\author{
Y. Ono, R. A. Ellis, Jr., A. C. Janos, F. M. Levinton; \\ R. M. Mayo! R. W. Motley, Y. Ueda and M. Yamada
}
Plasma Physics Laboratory, Princeton i niversity, Princeton, New Jersey 08544

Operation of the S-1 device in high current density $\left(j / n_{e} \geq 2 \times 10^{-14} \mathrm{~A} \cdot \mathrm{m}\right)$ regime has created high electron temperature spheromaks (50eV $\leq T_{\mathrm{e}} \leq$ $130 \mathrm{eV}$ ). The mechanisms and causes of the periodic relaxation events often observed in these hotter spheromak plasmas were made clear. Also, a rela: tionship between the MHD relaxation cycle and confinement characteristics was revealed for the first time. Resistive loss at the outer edge of the plasma causes a departure irom the initial force-free minimum-energy Taylor state to a MHD profile unstable to low-n ideal MHD modes; a relaxation event then returns the configuration to nearly a Taylor state.

- On assignment from JAYCOR, Torrance, CA 90503

'Purdue University, Weat Lafayette, IN 47906 
Plasma relaxation oscillations near the force-free, minimum-energy (Taylor) state, in both spheromaks and RFPs, have recently received markedly increased attention. The significant achievernents of this paper are identification of the mechanism of relaxation cycle around the Taylor state in high temperature spheromak plasnies and demonstration that the confinement characteristics of the spheromak are responsible for devistions from the Taylor state, causing relaxation phenomena which in turn affect confinement.

The minimum energy state of the force-free spheromak configuration is the Taylor state, ${ }^{1}$ in which $(\vec{j} \cdot \vec{B}) / B^{2}=\mu=$ constant throughout the plasma volume where $j$ is current density and $B$ is magnetic field strength. Actual spheromak plasmas are observed to be close to the Taylor state in the low temperature regime. ${ }^{2-8}$ In high temperature spheromak plasmas, deviations from a Taylor state ( $\mu=$ constant) are observed; in addition, a force-free configuration does not strictly permit a pressure gradient in the plasma since $\nabla p=\vec{j} \times \vec{B}=0$, where $p=$ pressure. The questions arive as to how much pressure (or pressure gradient) a spheromak in a near-Taylor state can maintain, and is the pressure limit due to MHD instabilities?

Relaxation oscillations were firat observed" in the S-1 Spheromak device on the global parameters. Spheromaks in CTX were observed" to deviate from the Taylor state and become unstable to low $n$-mode ( $n=$ toroidal mode number) instabilities, in agteement with theary, but did not exhibit relaxations. By measuring the radial magnetic field profile of spheromaks ja the CTCC device, ${ }^{7}$ sudden stepwise instabilities in the configuration could be explained by a periodic evolution from brosd stable profiles to peaked unstable profiles which then triggered a readjustment.

The present paper reveals the mechanism of relaxation event in S-1 spheromak plasmes. By measuring the detailed 2-D internal structure of the spheromak configuration, $q$ is directly measured; the stability of the configuration is thus determined and the cause of the relaxation is clearly revealed for the first time. Also for the first time, the effects of the relaxation events in spheromaks on confinement are identified in high temperature plasmas in the S.1 spheromak device by use of a multi-point Thomson scattering system yielding radial profiles of temperature and density. It was found that resistivity profiles (higher near the edge of the plasma) cause the spheromak to deviate from the Taylor state to one unstable to low-n modes. Resistivity then allows this configuration to undergo magnetic reconnection, letting the 
configuration relax back to the Taylor state. The relaxation is often accompanied by a degradation in confinement. Also, the relaxation involves a flux conversion process, important in both spheromaks and RFPs."

In the S-1 experiment, spheromak plasmas are produced by inductive transfer of toroidal and poloidal magnetic fux from a flux core which contains poloidal and toroidal windings, ${ }^{n, 3}$ The MHD stability against $r=1$ (tilt or shift) modes is maintained with the help of passive conductors consisting of a set of figure-eight coils ${ }^{2}$ and funnel-shaped conductors as shown in Fig.1.

Radial profiles of poloidal magnetic field $B_{p}$ and toroidal magnetic field $B_{1}$ are measured as a function of time with a magnetic probe array inserted into the plasma. By scanning the probe array discharge by discharge, 2-D magnetic field contours in the R-z plane were made to calculate poloidal flux $\Psi$, toroidal flux $\Phi$, and $q(\Psi)$ in the plasma. ${ }^{10,6}$ Globally coherent modes in the toroidal direction are monitored by a set of 16 external magnetic pick-up coils equally spaced in the toroidal direction at a distance of $60 \mathrm{~cm}$ from the midplane. The electron temperature $T_{t}$ and density $n_{t}$ profiles are measured by a seven channel Thomson scattering system. The messurement points are distributed every $7 \mathrm{~cm}$ radially on a line which is tilted by 25 degrees from the midplane, as shown in Fig.1.

Under a given operating condition, the relaxation events are somewhat reproducible. Discharges most frequently exhibit only one relaxation event although discharges with two or three events occur occasinally. By adjusting plasma parameters, the first (and only) relaxation event in a discharge was made very reproducible over many discharges, and these aie the discharges which are analyzed below.

Figure 2 shows the time evolution of the $T$. profile. The radial position in Fig.2 is defined as the distance along the laser bear:: path from the point where the beam crosse the midplane $(z=0 \mathrm{~cm}, R=47 \mathrm{~cm})$. After formation of the spheromak ( $t=300 \mu \mathrm{sec}$ ), the $T$. profile gradually peaks until $360 \mu \mathrm{sec}$. Peaking is caused by an increase in the temperature near the center of the plasma where the ohmic heating power is highest. A maximum $T_{e}$ of $\sim 75 \mathrm{eV}$ is reached at $t=360 \mu s e c$, when the peaking is most pronounced. This $T_{e}$ profile is consistent with a resistivity profile higher near the edge of the plasma. Since this data (Fig.2,3) was obtained with magnetic probes inserted into the plasma to measure field profiles at the same time, the maximum $T_{e}$ was lower ( $\sim 30 \%$ in this case) than was otherwise obtainable. 
Figure 3(a), (b) show the radial profiles of $B_{p}$ and $B_{l}$ on the midplane as a function of time. The $B_{p}$ profile near the magnetic axis ( $B_{\mathrm{p}}=0$ or $R \approx 40 \mathrm{~cm}$ ) gradually becomes steeper as time increases to 360 ssec. Based on $\nabla \times \vec{B}=\mu_{0} \vec{j}$, this steepening implies that $j_{t}$ is also peaking. The $B_{t}$ profile also shows a peaking during the same period; this is expected because the $B_{t}$ profile can be interpreted as representing the $j_{t}$ profile assuming an approximately constant $\lambda\left(\equiv j_{t} / B_{t}\right)$ profile. Within the resolution of the $T_{e}$ measurement, the peaking of $j_{l}$ and $T_{\varepsilon}$ show a very similar time evolution.

Hence, the resistivity profile produced by the strong heating in the core region causes the outer (poloidal) currents to decay faster than the inner (toroidal) currents. This, in turn, produces further preferential beating of the center region (positive feedback), while also causing a further departure from a Taylor state.

The $\Psi, \Phi$, and $\phi_{0}$ are shown in Fig.4(a) - (c). Imunediately after spheromak formation ( $t \approx 300 \mu s e c$ ), $\Psi / \Phi$ is about 2.7 and $q$ on axis, $q_{0}$, is 0.55 , which are expected values for the Taylor state. From $300 \mu \mathrm{sec}$ to $360 \mu \mathrm{sec}$, however, $\Psi / \Phi$ increases, indicating the development of poloidal-flux-rich configuration deviating from the Taylor state. The increase in $\Psi / \Phi$ is due to a preferential decay of $\Phi$ over $\Psi$ which is emphasized in the plot of $\Psi / \Psi$ - $\dot{\Phi} / \Phi$, the difference in decay rates. Only during this peaking period is the preferential decay of $\Phi$ over $\Psi$ so dramatic. Also during this peaking period, $q_{0}$ is decreasing. When $q_{0}$ falls below $1 / 2$, toroidal mode analysis indicates ${ }^{10}$ an $n=2, m=1$ mode suddenly develops and increases in amplitude. The amplitude of the $n=2$ (and all toroidal modes) is much lower early in the peaking phase.

The "peaking phase" is followed by a "broadening phase". From $360 \mu s e c$ to $390 \mu \mathrm{sec}$, the profiles of $B_{t}$ (and $j_{t}$ ) are clearly observed to broaden while the slope of $B_{p}$ near the magnetic axis decreases, again indicating a broadening of the $j_{t}$ profile. The polarity of $\dot{\Psi} / \Psi-\dot{\phi} / \Phi$ reverses, indicating a conversion of poloidal flux to torojdal flux ( $\dot{\Psi}<0, \dot{\phi}>0$ ). As this is the only time during decay that $\Psi / \Psi \cdot \Phi / \Phi$ switches polarity, it is a decisive indicator of flux conversion. The magnetic flux conversion brings the spheromak from the non-Taylor like state with excessive $\Psi$ to a Taylor-like state with the proper balance of $\Psi$ and $\Phi$ for $a$ given size and shape configuration. $\Psi / \Phi$ decreases to 3.0. Also $g_{0}$ returns to 0.55 . This relaxation event results in a $20 \%$ loss in total magnetic flux. 
The $T$. profile [Fig.2] broadens as a result of a sudden drop in central temperature. Density profile and density itself change very little during the relaxation. Possible causes of the drop in $T_{t}$ are a loss of high $T_{e}$ particles which are replaced by low $T_{e}$ particles of the edge region and/or a loss ef the central ohmic heating power, both of which are caused by the magnet: reconnection process. The former is consistent with the simulation of Sgro et al. ${ }^{1 t}$ which suggests that during the relaxation, the hot plasma near the original magnetic axis is expelled while a new axis, which is formed near the edge, brings in cold plasma. In the experiment, roughly $40 \%$ of the total thermal energy is lost. On a finer scale, high frequency ( $>50 \mathrm{kHz}$ ) magnetic fluctuations are observed to increase by a factor of $\sim 3$ to 5 during the relaxation, offering another loss channel.

In order to investigate the transport under the highest $T_{e}$ conditions, the magnetic probe was removed from the plasma. Under these conditions, a maximum peak temperature of over $100 \mathrm{eV}$ is obtained [Fig.4(d)] at about $350 \mu s e c$, just before the onset of relaxation. The energy confinement time, $\tau_{E}$, is calculated by comparing the energy input at the central core and the time evolution of the plasma kinetic energy assuming $T_{e}=T_{i}$. If $Z_{\text {anomalous }}$ is set between $3-5$, the maximum value of $\tau_{E}$ is between $50 \mu \mathrm{sec}$ and $100 \mu \mathrm{sec}$ in the middle of the peaking phase[Fig.4(e)].

The whole process of a relaxation cycle can be schematically represented as in Fig.5. In principle, the cycle described above can be repeated. In fact, multiple relaxation events are sometimes observed in S-1, giving the appearance of "sawtooth"-like phenomena. The behaviour of the decaying spheromak, and the number of relaxation events, depend on the magnetic Reynolds number $S$ and the resistivity profile, based on the simulation by Sgro et al." If $S$ is smaller than 500 , there is little resistivity gradient between the plasma's center and its edge so that the configuration decays self-similarly and no instability is encountered. For higher $S>2000$, the time between relaxations is predicted to become comparable to or greater than the configuration lifetime, so one expects to be able to observe only one or two relaxations, if any. For intermediate $S$, one predicts multiple relaxations; in practice, however, a relaxation reduces $S$ so that, again, only one or two relaxations at most may be expected. In $\mathrm{S}-1, S$ becomes as high as 3000 .

In summary, a "sawtooth" type relaxation cycle was observed both in the 
magnetic configuration and the pressure profile of S-1 spheromak discharges during the decay phase. It was found that the confinement characteristics of the spheromak were significantly affected by the relaxation phenomena, particularly in the broadening phase. In order to make the spheromak more viable to fusion reactor, it is essential to avoid these catastrophic relaxation phenoment by controlling current profile.

We acknowledge useful discussions with $\mathrm{T}$. $\mathrm{K}$. Chu on temperature and MHD propertiss. This work was supported by the U.S. Department of Energy under Contract No.DE-AC02-76-CH0-3073. 


\section{References}

'J. B. Taylor, Phỵs. Rer. Lett. 33.1139 (1974).

${ }^{2} \dot{G}$. C. Goldenbaum et al., Phys. Rev. Lett. 44, 393 (1980); T. R. Jarboe et al., Phys. Hev. Lett. 45, 1264 (1980).

${ }^{3}$ M. Yamada et al., Phys. Rev. Lett. 46, 188 (1981).

${ }^{4}$ G. W. Hart et a]., Phy's. Fluids 29, 1994 (1986).

5S. O. Knox et al., Phỵs. Rev. Lett. 56, 842 (1986).

${ }^{6}$ A. C. Janos, Phys. Fiuids 29, 3342 (1986).

${ }^{7}$ T. Lyama et al., Nucl. Fusion 27, 799 (1987).

${ }^{8}$ R. G. Watt and R. A. Nebel, Phys, Fluids 26, 1168 (1983); K. A. Werley et al., Phys. Fluids 28, 1450 (1985); V. Antoni and S. Ortolani, Phys. Fluids 30. 1489 (1987): R. B. Howell et al., Phys. Fluids 30, 1828 (1987).

${ }^{9} \mathrm{M}$. lamada et al., in Tenth International Conference on Plasma Flyysics and Controlled Nuclear Fusion Research, London. England, 1984, Vol.2, p.535.

${ }^{10}$ A. C. Janos et al., Phys. Flujds 28. 3667 (1985).

"A. G. Sgro. A. A. Mirin, and G. Mlarklin, Phỵs. Fluids 30, 3219 (1987). 


\section{Figures}

\section{FIG. 1. S-I device}

The dotted points on the laser beam path indicate the measuring points of Thomson scattering.

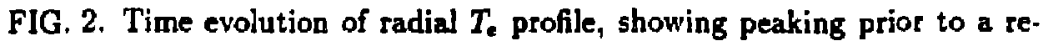
laxation event.

The radial position is defined by the distance along the laser beam path from the point where the beam crosses the midplane $(z=0 \mathrm{~cm}, \mathrm{R}=47 \mathrm{~cm})$.

FIG. 3. Radial profiles of (a) poloidal and (b) toroidal magnetic field on the midplane as a function of time

FIG. 4. Time evolution through a relaxation cycle during the decay phase of:

(a) $\Psi / \Phi$ (poloidal flux/toroidal flux)

(b) $\dot{\Psi} / \Psi-\dot{\Phi} / \Phi=$ Difference in decay rate of $\Psi$ and $\Phi$.

(c) $q$ value at the magnetic axis

(d) peak electron temperature $T_{e}$

(e) energy confinement time $\tau_{E}$

The thermal energies of the electrons and jons are assumed to be equal and $Z_{\text {anomalou }}$ is set to 3 . Therefore, if $Z_{\text {anomalous increases by a factor of }}$ $n, r_{e}$ roughly decreases by a factor of $n$.

FIG. 5. Schematic diagram of relaxation cycle 







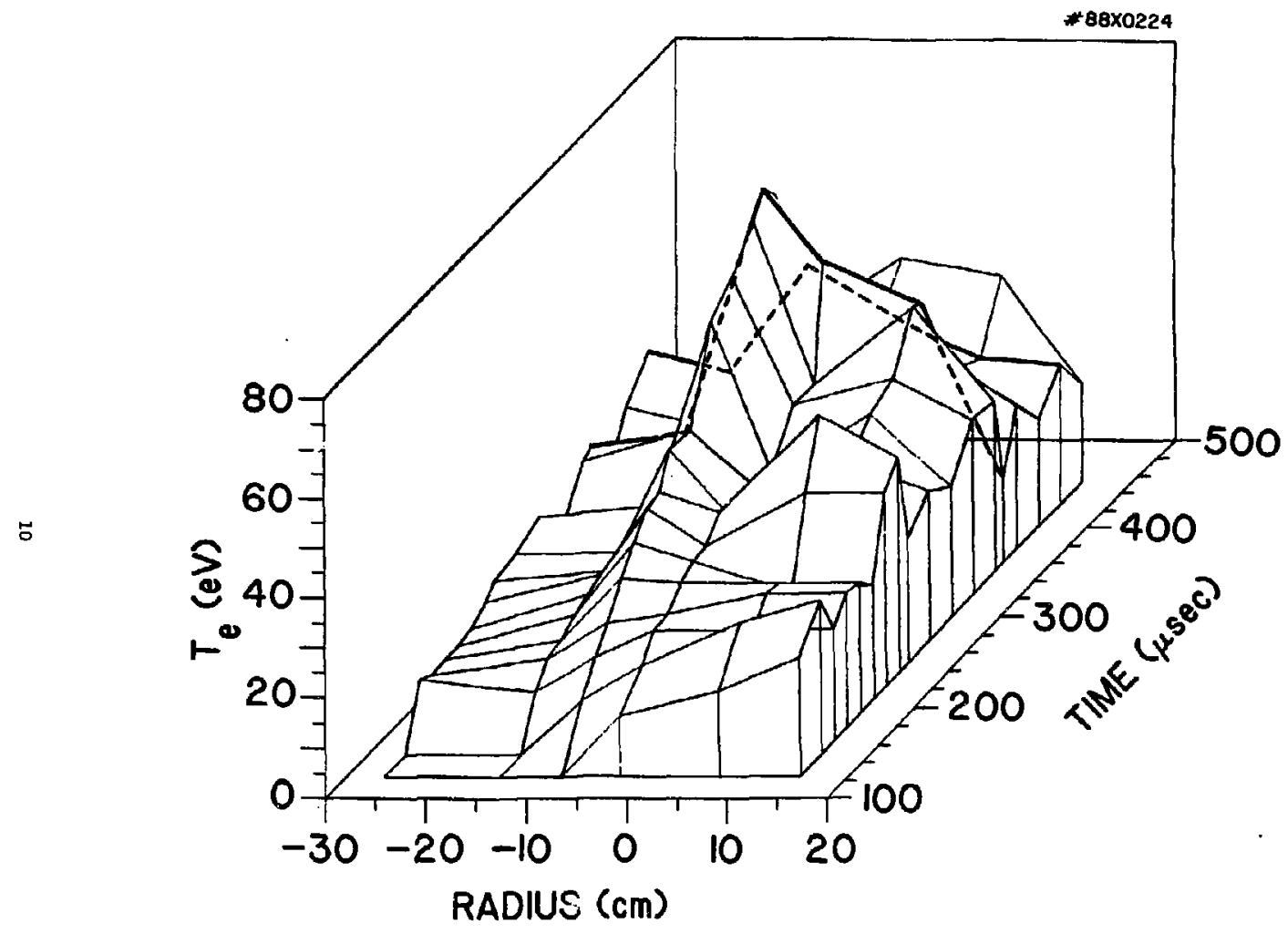



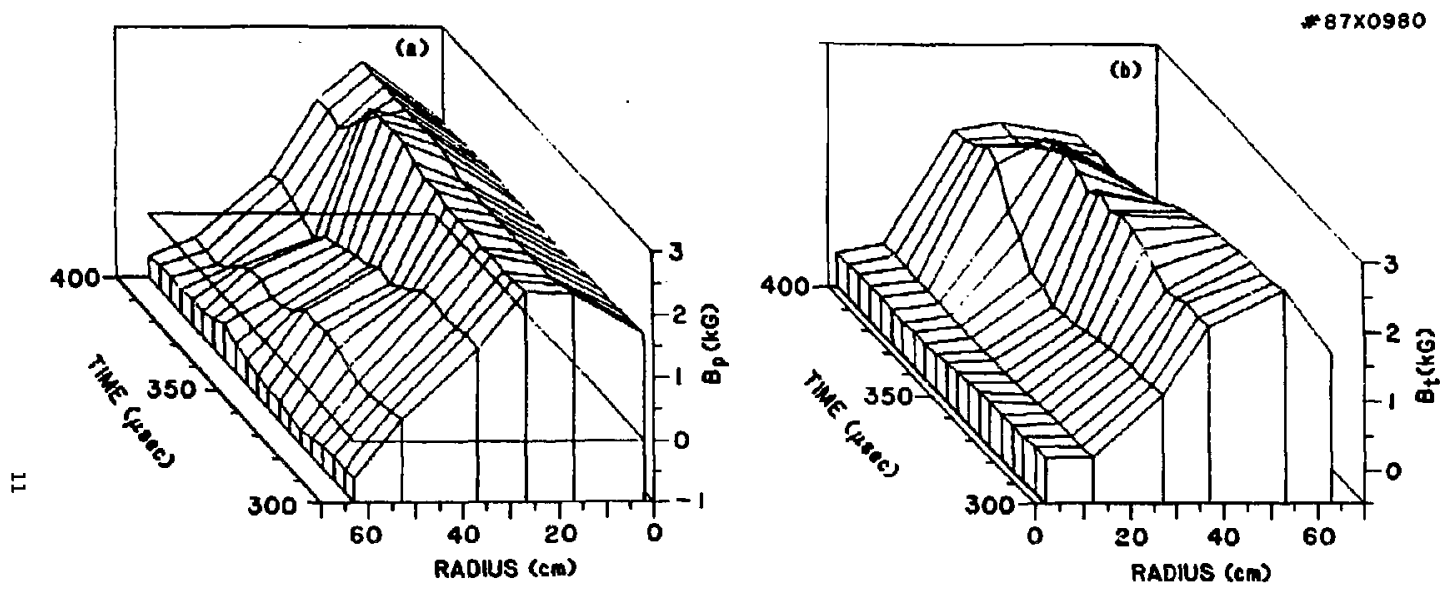

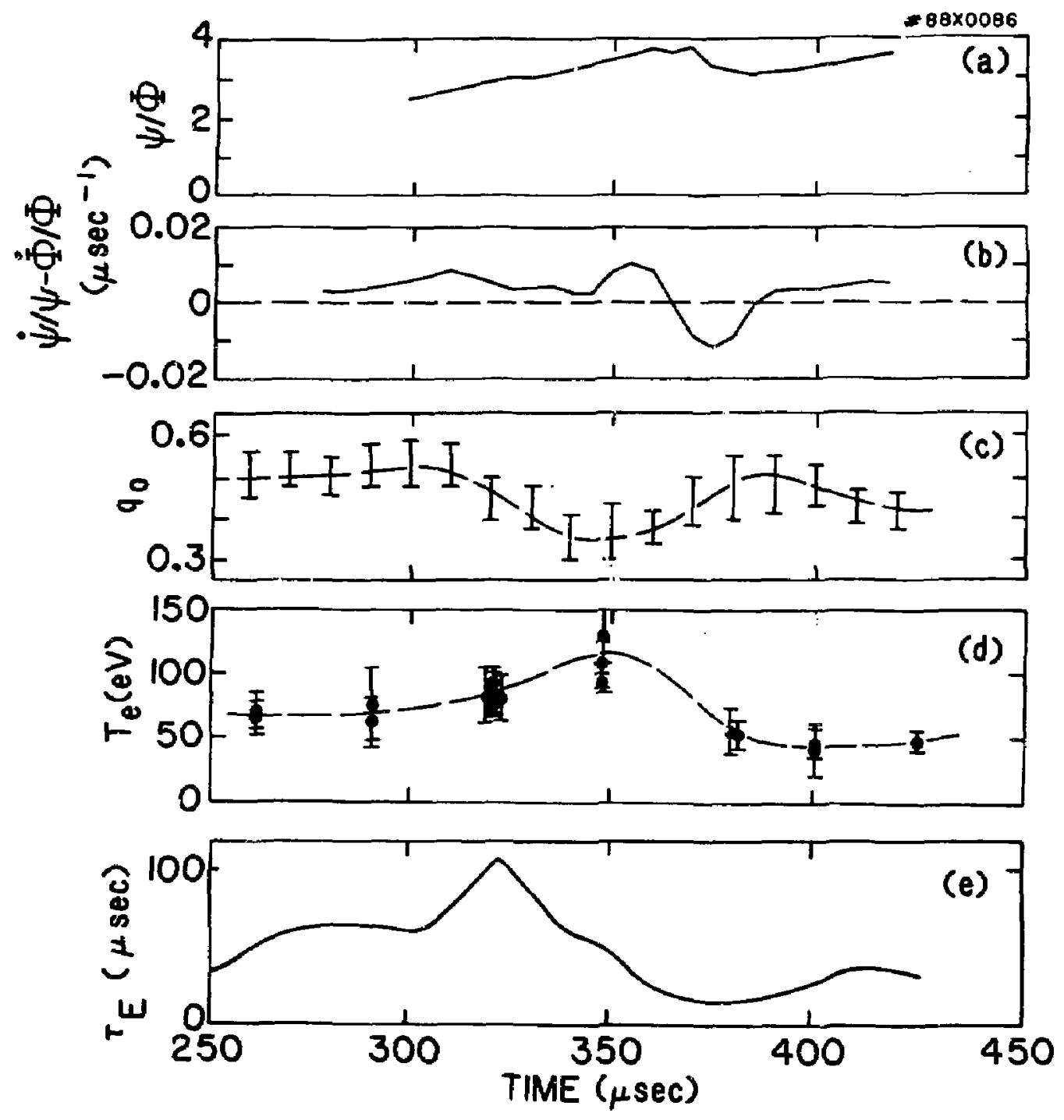

FIG. 4 


\section{\#88x0353}

Relaxation

- $m=1 \quad n=2$ Kink Mode

- Flux Conversion from $\Psi$ to $\Phi$

- Te $\downarrow$

$\left(\tau_{\mathrm{pf}}<\tau_{\mathrm{tf}}\right)$

w

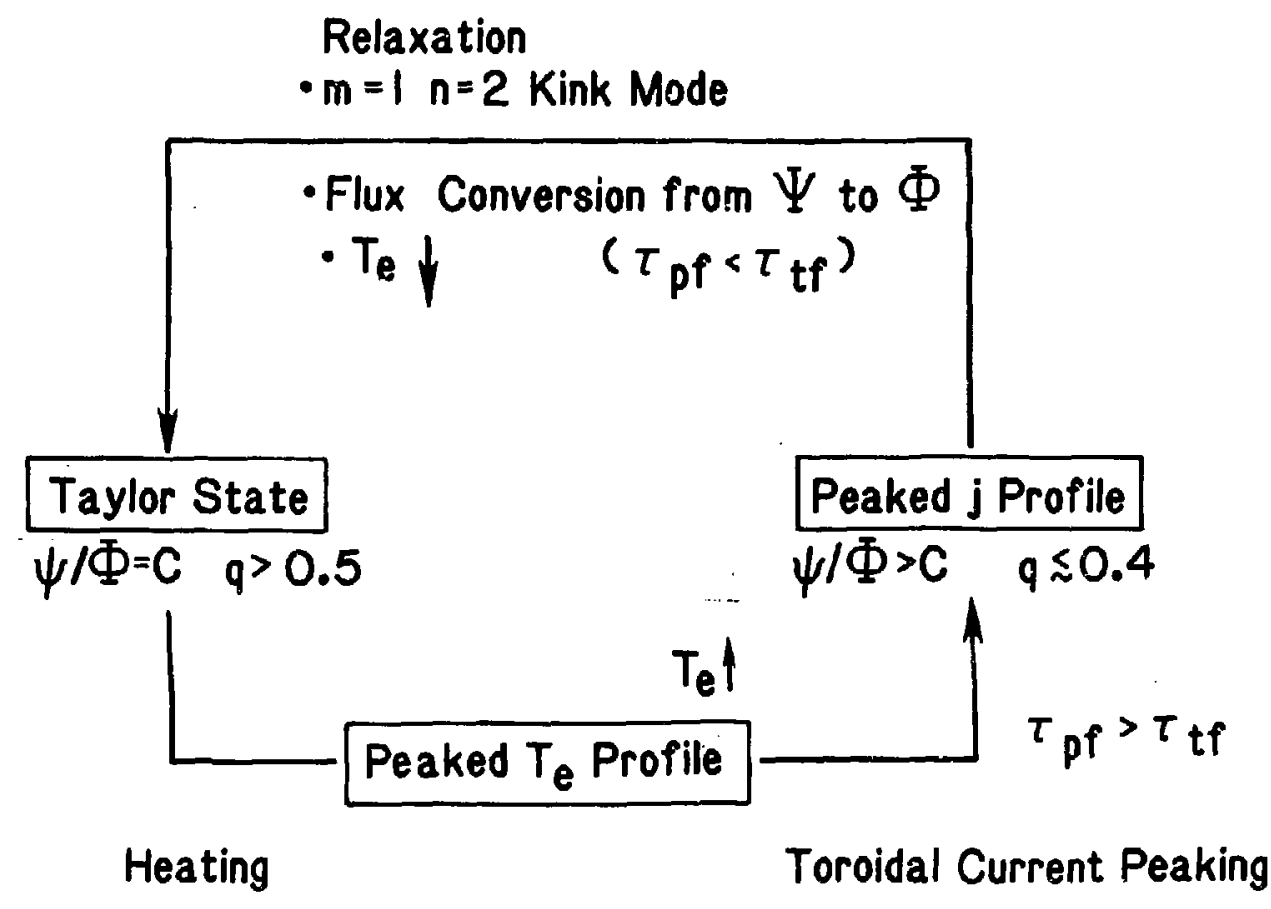

